\title{
Evaluation of Laboratory Methods to Measure Telomere Length in Cancer
}

\author{
Vikas Kumar Bhari ${ }^{1}$, Shreya Sharma ${ }^{1}$, Durgesh Kumar ${ }^{2}$, Surendra Kumar ${ }^{3}$, \\ Abhijeet Singh ${ }^{1}$ and Rajeev Mishra ${ }^{*}$
}

Department of Biosciences, Manipal University Jaipur, Rajasthan, India

${ }^{2}$ Department of Physiology, Government Medical College, Kannauj, Uttar Pradesh, India

${ }^{3}$ Department of Neurology, Rajendra Institute of Medical Sciences, Ranchi, Jharkhand, India

*Corresponding author

\begin{tabular}{|c|c|}
\hline & A B S T R A C T \\
\hline Keywords & \multirow{4}{*}{$\begin{array}{l}\text { Telomere comprises of TTAGGG repeats and its lack of functionality results in } \\
\text { promoting cancer through driving genetic instability. It is evident that they protect } \\
\text { chromosomal ends and conserve our genetic information. This review discusses } \\
\text { various laboratory methods of telomere length measurement. Understanding more } \\
\text { about how these methods work and can be modified, they can be universalized in } \\
\text { terms of their use all over the globe. Regardless of whether telomere length } \\
\text { quantification has a direct or indirect association with cancer progression// } \\
\text { prevention, its assessment has been shown to hold promise for better prognosis } \\
\text { and reliable biomarker which allow the better assessment in early identification } \\
\text { and therapeutic responses. }\end{array}$} \\
\hline $\begin{array}{l}\text { Telomere, } \\
\text { Biomarker, FISH, } \\
\text { qPCR }\end{array}$ & \\
\hline Article Info & \\
\hline $\begin{array}{l}\text { Accepted: } \\
\text { 08 June } 2020 \\
\text { Available Online: } \\
10 \text { July } 2020\end{array}$ & \\
\hline
\end{tabular}

\section{Introduction}

Telomere length is an important measure to discuss if one is trying to understand about the telomere biology. The standard method worldwide for telomere length detection is southern blot analysis, although the process is relatively slow and tiresome. New techniques are developed to overcome limitations and get better results. Fluorescence in situ hybridization (FISH) helps in calculating length on the basis of number of the telomeric repeats. Improved modifications of FISH like quantitative FISH (Q-FISH) and quantitative flow cytometry (Q-FISH ${ }^{\mathrm{FCM}}$ ) are beneficial for accurate length measurement of telomere (Aubert, Hills, and Lansdorp 2013). Here we discuss several popular methods of telomere length measurement.

\section{Laboratory methods used in clinics to measure telomere length}

Regardless of whether telomere length quantification has a direct or indirect association with cancer progression/ prevention, its assessment has been shown to hold promise for better prognosis and reliable 
biomarker which allow the better assessment in early identification and therapeutic responses. Recent advances in technology have made it possible to identify different robust and reproducible TL measurement techniques. Therefore, understanding the benefits and drawbacks of TL measurement methods is important is necessary for comparing the results published by different investigative teams, as well as designing experiments by the researcher. We summarize the classical and widely acceptable strategies used in telomere length measurement which include Q-PCR (Quantitative Polymerase Chain Reaction),

TRF (Terminal Restriction Fragment) analysis, a variety of Q-FISH methods, STELA (Single TElomere Length Analysis) and TeSLA (Telomere Shortest Length Assay) as described in Figure 1. Here we are discussing major methods developed and being used as pieces of evidence by various published research to measure telomere lengths (TLs). We will also be discussing their advantages and limitation associated with these techniques.

\section{Q-FISH methods}

Q-FISH is a very popular technique used to measure the telomere length in a single or specific cell population. This technique provided better specificity, increased sensitivity, and resolution. Traditional QFISH is called Q-FISH on metaphases. Metaphase chromosomes are hybridized with telomere repeat specific (CCCTAA) n probes. Usually a synthetic peptide nucleic acid (PNA) probe is used as its neutral backbone to provide better hybridization efficiency than traditional DNA probes (Egholm et al., 1993). Standard Q-FISH image uses double staining, one fluorophore to stain and probe with telomere and another for chromosome such as 4,6-diamidino-2-phenylindole dihydrochloride (DAPI) (Krejčí and Koch 1998).
Image is then processed, analyzed and results are developed into histograms showing telomere length frequencies. Q-FISH allow one to differentiate telomere length between chromosomes and that may connect to chromosome instability. Telomere-free ends or chromosomes with critically short telomere can also be identified using this technique which can be of great utility as short telomeres are associated with age- related disease risk.(Vera et al., 2012) This technique is however labor-intensive, time consuming and requires pre-requisite knowledge about chromosome banding patterns which make it less preferable for large population studies.

Interphase Q-FISH was developed recently as Q-FISH on metaphases can only detect telomere length of proliferating cells. Interphase Q-FISH technique can detect telomere length in tissue sections or other cells of non-proliferative origin such as differentiated or senescent cells (GonzálezSuárez et al., 2000). Telomere signal obtained in this technique is not from individual but from a pool of some telomeres knowns as "telomere spots" due to which telomere free ends are not possible to detect (Vera and Blasco 2012). Interphase Q-FISH is less labor intensive and time consuming because of the recent advancement known as highthroughput (HT) which uses PNA probes and hybridization process like in metaphase QFISH technique but it uses interphase cells for process and HT microscopy instruments are used. HT Q-FISH can be used extensively for population studies (Canela et al., 2007).

Flow-FISH method, another modification of Q-FISH has been used extensively in disease research. Flow-FISH combines principles of metaphase FISH and fluorescence-activated cell sorting (FACS). Cells are not fixed but cell suspension is first hybridized with PNA probe specific to telomere repeats and then cells are passed from lasers (Rufer et al., 
1998). Mean amount of fluorescence is detected and used to quantify an average telomere length. It is a powerful technique in which cells can be sorted out based on the size, granularity and labelling of antibody.

\section{TRF analysis}

Since the sequence TTAGGG $_{n}$ is highly conserved in mammals, this technique was developed using TTAGGG $_{n}$ specific probes detected by southern blotting (Kimura et al., 2010; Lansdorp et al., 1996). Genomic DNA is digested using a mixture of commonly used restriction enzymes into small fragments. Telomeric and the sub-telomeric regions are not cleaved and terminal restriction fragment remains intact as they do not contain the restriction site of the chosen restriction enzymes. Based on the size, telomere from all the chromosomes are separated using agarose gel electrophoresis and the smears of telomere are detected by southern blotting or in-gel hybridization using telomeric sequence probes, after this intensity is quantified to obtain average telomere length by comparing smear with DNA ladder of known size (Kimura et al., 2010; Lansdorp et al., 1996). This method is considered as a "gold standard" for its use in measuring telomere length but there are many drawbacks to this method. Firstly large amount of DNA is required which hinders its use where cells or DNA is minimal. Secondly, apart from telomeric repeats, this technique also doesn't cleave sub-telomeric region and the addition of this region results in an overestimation of telomere length. Telomere and sub-telomere region also contain many polymorphisms which can give unpredictable different results. Use of different restriction enzymes in different labs can pose problems in its comparability of results and its clinical utility. Most important drawback, however, is that telomere length below the threshold length cannot be detected as it is a hybridization based method and it is evident that shortest telomeres are only responsible for chromosome instability and cancer progression (Hemann et al., 2001).

\section{Telomere shortest length assay (TeSLA)}

Telomere shortest length assay (TeSLA) is a new advancement in methods of single telomere length measurements. TeSLA-Ts comprising nucleotides of $\mathrm{C}$-rich repeats of telomere are ligated to the extracted genomic DNA at the 3' end overhang and digested with the sets of restriction enzymes. Double stranded TeSLA adapters are designed and ligated to both ends of telomere and genomic DNA fragments obtained after digestion. PCR is performed after the adapter ligation (Lai et al., 2017). TeSLA is much more sensitive, efficient, and specific than U-STELA in regards of telomere length measurement. This technique is not biased towards long telomere as it can detect both shortest telomere as short as $1 \mathrm{kB}$ and longer telomeres up to $18 \mathrm{kB}$.

\section{Single telomere length analysis (STELA)}

There was a need to develop a technique which can detect individual telomere length in contrast to TRF and qPCR-based assays which detect average telomere length of the sample analyzed. Need of this type to technique is logical as it has been well established that it is the single shortest telomeres which are responsible to trigger DNA damage responses and eventually cause replicative senescence (Fumagalli et al., 2013; Hemann et al., 2001). Thus crucial information about the shortest telomere can prove to be a crucial biomarker for cancer.

Single telomere length analysis (STELA) is a PCR and ligation-based assay which makes use of a linker or 'telorette' having combination of telomere sequence (TTAGGG) and 20 nucleotides of the non- 
specific complementary tail. It is annealed to the 3' overhang of telomere end. This linker is then ligated to the complementary $5^{\prime}$ end of chromosome. Primers are designed for this specific telomere tail and telomere is amplified (Baird et al., 2003). One major drawback of this technique is that this technique has been restricted to telomere length measurement of only few chromosomes (XpYp, 2p, 11q, and 17p) as it is a primer-based assay. This problem was solved by the emergence of Universal STELA (U-STELA) which measures any critically short telomere regardless of the position of the chromosome (Bendix et al., 2010), But this assay is unable to detect telomere length beyond 8kB (Bendix et al., 2010).
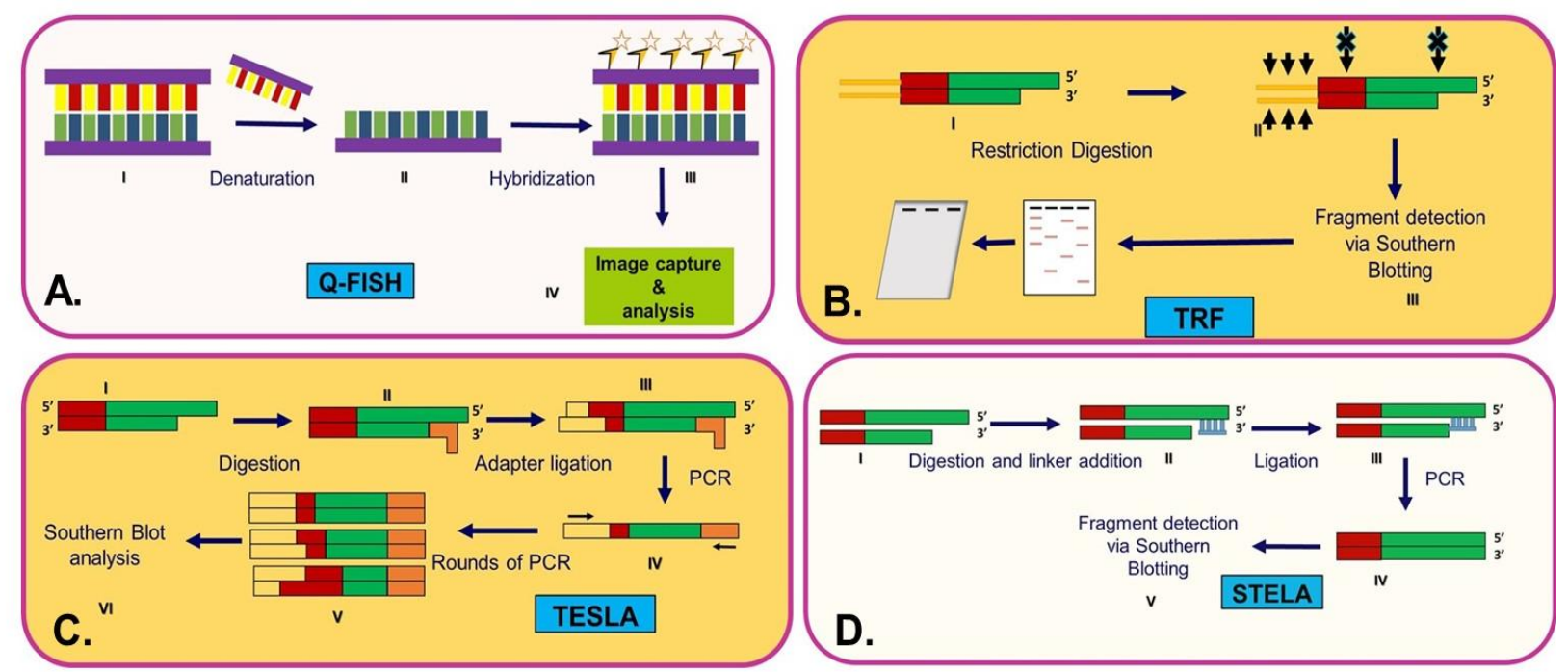

\section{Telomere length measurement methods}

Figure.1 Mechanism of different telomere length measurement methods

Fig.1 showing flow chart based mechanisms of Quantitative Fluorescent in situ Hybridization (Q-FISH), Terminal restriction fragment (TRF), Single Telomere Length Analysis (STELA) and Telomerase Shortest Length Assay (TeSLA). (Fig.A) Q-FISH is a cell based technique which is based on the traditional FISH principle. After denaturation of ds DNA, peptide nucleic acid (PNA) oligonucleotides are added to target telomere sequences using fluorescent microscopy and image is analyzed and length is determined.

(Fig.B) TRF method uses southern blotting based method to quantify telomere length. This method uses a mixture of restriction enzymes which create small fragments of
DNA, leaving telomere and sub-telomere DNA intact. Upon size separation and transfer, telomere length is quantified using known DNA ladder.

(Fig.C) In TeSLA method, similarly to STELA, a linker (TeSLA Ts) are ligated to the telomere overhang and then restriction enzyme cocktail is used to digest DNA. Double stranded TeSLA adapters are attached to the digested DNA and telomeric DNA is amplified using PCR. Adapted by (Lai et al., 2017). (Fig.D) STELA makes use of a linker, annealed to the 3' overhang and ligated consequently to the 5' end of telomere end. Linker specific primers amplify the telomere and length is calculated. 
Quantitative polymerase chain reaction (qPCR) method

The major challenge in telomere biology is the need of large quantities of DNA and longer incubation time to evaluate telomere lengths. This hurdle was overcome by Richard Cawthon, who introduced a robust and reliable method to quantify telomere length by polymerase chain reaction (PCR)based analysis (Campisi 2003). This method includes the qPCR amplification of genomic DNA sequence of interest up to 40 cycles using specifically designed primers for Telomere (T) and Single copy (S) genes. Typically, in qPCR, the input DNA sequence is amplified using fluorophore (which emits a fluorescent signal) that intercalates with double stranded DNA (dsDNA) (i.e., SYBR green dye). The ratio of amplified product of telomere and single-copy gene (36B4) i.e. T/S was calculated to yield a value that correlates with relative telomere length (RTL). This method is fast, cost effective, less amount of DNA requirement $(50 \mathrm{ng})$ and use in high throughput studies makes this method as widely versatile method to measure telomere length. However, over a period of time this method was criticized having certain limitations in measurement precision (pipetting, etc.) which was an obstacle to get reproducibility in obtained results even in wells/tubes.

To overcome these limitations, Cawthon, in his follow up paper in 2009 explained a monochrome multiplex qPCR method that enables the measurement of both $\mathrm{T}$ and $\mathrm{S}$ signal in same reaction (Cawthon 2009). Principle given behind this method was that telomere signals will be collected in early PCR cycles when single copy signal will be only at the baseline, and then later PCR cycles will collect only single copy genes as the temperature set is will fully melt the telomere product. This works as the telomere signal is much more abundant than single copy gene. This modification in previously described method improves the qPCR approach significantly.

Telomere length measurement as a clinical marker is coming in light. There is accumulating evidence from many types of studies such as large cohort and systematic analysis showing telomere length is correlated with all-cause mortality and cancer risk. However, the use of different methodologies to measure telomere length and source of DNA results in large coefficients of variation which are unacceptable in the clinical setting. There is a need to develop standard methods, protocols, and reagents to achieve the clinical significance of telomere length as a clinical biomarker.

\section{Acknowledgement}

The work was supported in part by Start-up Research Grant (SRG/2019/001049), from the Science and Engineering Research Board (SERB), Government of India and Enhanced Seed grants EF/2018-19/QE04-11 (to R.M.) from Manipal University Jaipur, Rajasthan, India is gratefully acknowledged.

\section{References}

Aubert, Geraldine, Mark Hills, and Peter M. Lansdorp. 2013. "Assessment of the Available Technologies and Tools." 730(604):59-67.

Baird, Duncan M., Jan Rowson, David WynfordThomas, and David Kipling. 2003. "Extensive Allelic Variation and Ultrashort Telomeres in Senescent Human Cells." Nature Genetics 33(2):203-7.

Bendix, Laila, Peer Bendix Horn, Uffe Birk Jensen, Ivica Rubelj, and Steen Kolvraa. 2010. "The Load of Short Telomeres, Estimated by a New Method, Universal STELA, Correlates with Number of Senescent Cells." Aging Cell 9(3):383-97. Campisi, Judith. 2003. "Cellular Senescence." 
Chromosomal Instability and Aging: Basic Science and Clinical Implications 30(10):29-50.

Canela, Andrés, Elsa Vera, Peter Klatt, and María A. Blasco. 2007. "High-Throughput Telomere Length Quantification by FISH and Its Application to Human Population Studies." Proceedings of the National Academy of Sciences of the United States of America 104(13):5300-5305.

Cawthon, Richard M. 2009. "Telomere Length Measurement by a Novel Monochrome Multiplex Quantitative PCR Method." Nucleic Acids Research 37(3):1-7.

Egholm, M., O. Buchardt, L. Christensen, C. Behrens, S. M. Freier, D. A. Driver, R. H. Berg, S. K. Kim, B. Norden, and P. E. Nielsen. 1993. "PNA Watson-Crick." Nature 365:566-68.

Fumagalli, Marzia, Francesca Rossiello, Michela Clerici, Sara Barozzi, Jessica M. Kaplunov, Gabriele Bucci, Miryana Dobreva, Valentina Matti, Christian M. Beausejour, Utz Herbig, and Maria Pia Longhese. 2013. "NIH Public Access." 14(4):355-65.

González-Suárez, Eva, Enrique Samper, Juana M. Flores, and María A. Blasco. 2000. Telomerase-Deficient Mice with Short Telomeres Are Resistant to Skin Tumorigenesis. Nature Genetics 26(1):114-17.

Hemann, Michael T., Margaret A. Strong, Ling Yang Hao, and Carol W. Greider. 2001. The Shortest Telomere, Not Average Telomere Length, Is Critical for Cell Viability and Chromosome Stability. Cell 107(1):67-77.
Kimura, Masayuki, Rivka C. Stone, Steven C. Hunt, Joan Skurnick, Xiaobin Lu, Xiaojian Cao, Calvin B. Harley, and Abraham Aviv. 2010. "Measurement of Telomere Length by the Southern Blot Analysis of Terminal Restriction Fragment Lengths." Nature Protocols 5(9):1596-1607.

Krejčí, Kateřina, and Jørn Koch. 1998. "Improved Detection and Comparative Sizing of Human Chromosomal Telomeres in Situ." Chromosoma 107(3):198-203.

Lai, Tsung Po, Ning Zhang, Jungsik Noh, Ilgen Mender, Enzo Tedone, Ejun Huang, Woodring E. Wright, Gaudenz Danuser, and Jerry W. Shay. 2017. "A Method for Measuring the Distribution of the Shortest Telomeres in Cells and Tissues." Nature Communications 8(1):1-13.

Lansdorp, Peter M., Nico P. Verwoerd, Frans M. Van De Rijke, Visia Dragowska, Marie Térèse Little, Roeland W. Dirks, Anton K. Raap, and Hans J. Tanke. 1996. Heterogeneity in Telomere Length of Human Chromosomes. Human Molecular Genetics 5(5):685-91.

Rufer, Nathalie, Wieslawa Dragowska, Gayle Thornbury, Eddy Roosnek, and Peter M. Lansdorp. 1998. "Flow Cyton1Etry." 743-47.

Vera, Elsa, and Maria A. Blasco. 2012. "Beyond Average: Potential for Measurement of Short Telomeres." 4(6):379-92.

Vera, Elsa, Bruno Bernardes de Jesus, Miguel Foronda, Juana M. Flores, and Maria A. Blasco. 2012. The Rate of Increase of Short Telomeres Predicts Longevity in Mammals. Cell Reports 2(4):732-37.

\section{How to cite this article:}

Vikas Kumar Bhari, Shreya Sharma, Durgesh Kumar, Surendra Kumar, Abhijeet Singh and Rajeev Mishra. 2020. Evaluation of Laboratory Methods to Measure Telomere Length in Cancer. Int.J.Curr.Microbiol.App.Sci. 9(07): 672-677.

doi: https://doi.org/10.20546/ijcmas.2020.907.077 